\title{
ReMed offre un soutien dans des situations de crise personnelle
}

\author{
Le réseau ReMed conseille et accompagne les médecins dans des moments critiques, \\ par exemple lorsqu'une crise personnelle risque d'avoir des conséquences sur leur \\ activité professionnelle quotidienne.
}

Jürg Beutler

Service de communication de la FMH

\section{Expérience personnelle}

Un médecin de famille âgé de 59 ans qui travaille dans un cabinet de groupe d'une petite ville et est père de deux enfants adultes parle de sa dépression:

Ce fut un coup très dur: après 22 ans de mariage, ma femme m'a annoncé qu'elle aimait un autre homme et qu'elle voulait me quitter. J'ai sombré dans une crise profonde. Il est vrai que mon métier occupait une grande place dans ma vie, mais ma femme avait également ses propres activités. Cette manière de vivre nous a convenu pendant longtemps et voilà que tout à coup, ça n'allait plus. Rétroactivement, je sais bien que nous nous étions éloignés l'un de l'autre. Ma femme m'a reproché de ne pas avoir été là après son grave accident de la circulation, c'est certainement vrai, mais je ne l'ai pas réalisé à l'époque.

La réaction de nos enfants fut particulièrement douloureuse. Pleins de reproches, ils ne nous ont pas adressé la parole pendant des semaines, et ils ne nous
Ce réseau de soutien conseille et accompagne les médecins en situation de crise. Lors d'une prise de contact, ReMed réagit dans les $\mathbf{7 2}$ heures et examine la situation et les démarches individuelles possibles. ReMed est tenu au secret médical. Avez-vous besoin d'aide? Ou un médecin de votre entourage?

Alors n'hésitez pas à prendre contact avec ReMed: Ligne d'assistance 24h/24: 08000 73633, info@swiss-remed.ch, www.swiss-remed.ch comprennent toujours pas aujourd'hui. Et pourtant nous avons été et sommes aujourd'hui encore de bons parents. La situation était intenable et j'ai quitté la maison le plus rapidement possible, et j'ai demandé le divorce. Comment ai-je réussi à sortir de cette crise? J'ai la chance de ne pas avoir d'inhibitions envers la psychiatrie et j'avais déjà fait appel à un professionnel auparavant lors de déprimes légères. J'ai donc pris contact avec un spécialiste qui me connaissait. J'ai ensuite immédiatement informé ma collègue du cabinet. Ils m'ont soutenu sans s'en mêler, ce qui m'a beaucoup aidé. J'ai également pu compter sur mes amis et mon rythme de travail bien établi m'a également aidé. J'ai ainsi pu gérer cette crise existentielle sans que mon travail de généraliste en pâtisse.
Correspondance:
Dr Michael Peltenburg Spécialiste FMH en médecine générale michael.peltenburg@hin.ch
Je ne peux donc que conseiller à mes collègues de chercher rapidement de l'aide lorsqu'ils traversent une période difficile. Aujourd'hui, ils peuvent même s'adresser à ReMed.

\section{Entretien avec le psychiatre Peter Birchler}

M. Birchler, vous faites partie du comité de direction de ReMed et avez, en tant que psychiatre, des contacts directs avec les personnes concernées. Est-ce particulier pour vous qu'un confrère vienne consulter?

La situation est particulière dans le sens où je veux aussi prendre en compte l'activité professionnelle du patient. En consultation, je suis face à un patient et collègue en situation de crise qui souhaite être entendu et soutenu. L'élaboration commune d'un objectif thérapeutique englobe en règle générale aussi les compétences et expériences professionnelles du patient. J'essaye notamment de lui montrer que ses difficultés personnelles ne doivent a priori pas être considérées comme de l'incompétence professionnelle. Le fait que nous venions du même contexte professionnel peut aider à établir une relation de confiance entre patient et thérapeute. En outre, il va de soi que le respect strict du secret professionnel vaut également pour un confrère.

Pensez-vous qu'il est plus difficile pour les médecins de demander de l'aide?

D'après mon expérience, oui. Nous autres médecins, nous pensons encore toujours que nous devons gérer les crises nous-mêmes et que nous n'avons pas de «vrais» problèmes. Pour beaucoup d'entre nous, demander de l'aide est une marque de faiblesse et non une preuve de compétence et de prise de responsabilités. J'estime donc qu'il est primordial que les médecins puissent, grâce à ReMed, dans un contexte professionnel de plus en plus difficile, compter sur une compréhension collégiale et une aide professionnelle. 